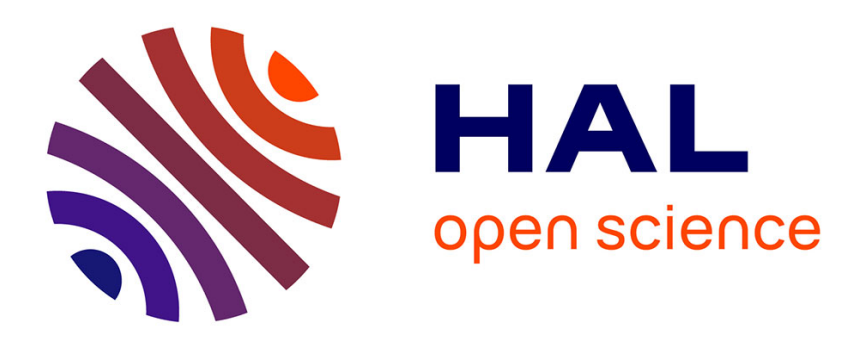

\title{
Equilibrium unzipping at finite temperature
}

Hudson Borja da Rocha, Lev Truskinovsky

\section{To cite this version:}

Hudson Borja da Rocha, Lev Truskinovsky. Equilibrium unzipping at finite temperature. Archive of Applied Mechanics, 2019, 89 (3), pp.535-544. 10.1007/s00419-018-1485-4 . hal-02415217

\section{HAL Id: hal-02415217 \\ https://hal.science/hal-02415217}

Submitted on 17 Dec 2019

HAL is a multi-disciplinary open access archive for the deposit and dissemination of scientific research documents, whether they are published or not. The documents may come from teaching and research institutions in France or abroad, or from public or private research centers.
L'archive ouverte pluridisciplinaire $\mathbf{H A L}$, est destinée au dépôt et à la diffusion de documents scientifiques de niveau recherche, publiés ou non, émanant des établissements d'enseignement et de recherche français ou étrangers, des laboratoires publics ou privés. 


\title{
Equilibrium unzipping at finite temperature
}

\author{
Hudson Borja da Rocha ${ }^{1,2, *}$ and Lev Truskinovsky ${ }^{2, \dagger}$ \\ ${ }^{1}$ LMS, CNRS-UMR 7649, Ecole Polytechnique, \\ Université Paris-Saclay, 91128 Palaiseau, France \\ ${ }^{2}$ PMMH, CNRS - UMR 7636 PSL-ESPCI, 10 Rue Vauquelin, 75005 Paris, France
}

(Dated: December 17, 2019)

\begin{abstract}
We study thermally activated unzipping, which is modeled as a debonding process. The system is modeled as a parallel bundle of elastically interacting breakable units loaded through a series spring. Using equilibrium statistical mechanics, we compute the reversible response of this mechanical system under quasi-static driving. Depending on the stiffness of the series spring, the system exhibits either ductile behavior, characterized by noncooperative debonding, or brittle behavior, with a highly correlated detachment of the whole bundle. We show that the ductile to brittle transition is of the second order and that it can also be controlled by temperature.
\end{abstract}

\section{INTRODUCTION}

In engineering applications, fracture is usually modeled as an athermal, irreversible process [1]. Even though thermally activated crack nucleation and propagation have been observed experimentally [2-4] and modeled as a kinetic process [5-8], the importance of temperature in non-extreme conditions is only marginal because of the enormous timescales involved in thermally induced fracture [9-11].

The situation is drastically different in biological applications where, for instance, cell adhesion is characterized by low binding energies of the order of $k_{B} T$, which is $\sim 4.1 \mathrm{pN} \mathrm{nm}$ at physiological temperatures $T \sim 300 \mathrm{~K}$. Such weak bonding can be disrupted by thermal activation leading to finite lifetimes of the bonds [12-14]. As a result, the temperature can be an important factor controlling the debonding processes; in particular, it plays a crucial role in the zipping-unzipping phenomena involved in the functioning of biological macromolecules [15-18].

Recent developments in force spectroscopy allowed accurate measurement of thermally induced unzipping for a broad variety of 'breakable' biological systems from two stranded DNA [19] to adhering cells $[20,21]$. In these experiments, one often observes intermittent response implying the presence of cooperative breaking events. Similar phenomena have also been detected during the detachment of cellular aggregates [22] and micropipette force-probing of single-cell adhesions [23].

Statistical mechanics has been used in the studies of various minimal models of thermal fracture $[3,24-29]$. One is often interested in understanding how the external loading affects the stability of the bonded state [30] and how it influences its lifetime [12]. The typical approach involves the study of stochastic dynamics of elastically interacting binders [31] with usually phenomenologically postulated dependence of the attachment and detachment rates on the applied load [32, 33]. The simplest model of this type is a parallel assembly of $\mathrm{N}$ binders interacting through a rigid backbone which introduces infinitely long-range, mean-field-type interactions. Such models have been very successful in the studies of molecular denaturation and unzipping and also advanced our understanding of actomyosin contraction [34, 35] and cell adhesion [36-38].

We recall that in the theory of biological adhesion, the dissociation of a single binder is perceived as a one-dimensional motion of a Brownian particle in a viscous environment. Bell's classical

\footnotetext{
* hudson.borja-da-rocha@polytechnique.edu

† lev.truskinovsky@espci.fr
} 
approach to binding kinetics, which is fundamentally similar to the Huxley-Simmons model of muscle contraction [39], takes into account in the most basic form the effect of applied forces on the rate of dissociation of a single binder [12]. It was later realized that the strength of a bond also depends on the rate at which the bond is loaded $[14,40]$. The dissociation was modeled as an escape from a metastable energy well and treated within the framework of Kramers's rate theory $[41,42]$. More recently, a stochastic version of Bell's model for a system of elastically interacting binders was used to show that the nonlinear rebinding kinetics results in collective unzipping and macroscopic bi-stability [30, 38].

In this paper, we study a microscopic model of thermal fracture taking place in quasi-static equilibrium conditions. Our starting point is the democratic fiber bundle model (FBM) [43, 44] which we augment by adding seemingly innocent internal (series to the binders) and external (series to the bundle) springs. For analytical transparency, we also assume that each fiber has a piecewise quadratic elastic energy. We neglect the quenched disorder in the breaking thresholds and instead expose the system to the annealed disorder provided by an equilibrium thermostat. The system can be studied in soft and hard devices. We recall that in mechanics, a machine that prescribes displacements on the boundary is called a hard loading device; instead, a machine that prescribes surface tractions is called a soft loading device [45]. We also recall that in the conventional version of FBM, the system is exposed to a constant force (loaded in a soft device) and the thermal equilibrium behavior is trivial because even at zero temperature the ground state is formally absent. The role of the loading modality (force or displacement control) on the outcome of the equilibrium stretching of macromolecules was discussed in [46]; for similar effects on molecular unfolding see [35].

More specifically, we consider a collection of $N$ breakable units connected in parallel between two rigid bars. When an individual unit breaks, the load is redistributed equally between the surviving units. The implied mean-field-type coupling allows individual binders to interact and opens a possibility for cooperative behavior. The resulting model is analytically transparent, and we computed its equilibrium thermodynamic properties explicitly. Our main technical result is the computation of the equilibrium free energy showing the second-order nature of the temperatureinduced brittle-to-ductile transition. Our study complements the analysis of a closely related actomyosin system represented by a parallel bundle of bi-stable units loaded in a soft device [47]. In the context of cell adhesion and cell motility, the out-of-equilibrium behavior of similar systems has been studied previously in [48-50].

The paper is organized as follows. In Sec. 2 we study the equilibrium mechanical response of a single elastic binder and show that such element loses its snap-through response at any nonzero temperature. In Sec. 3 we extend this analysis to the case of a parallel bundle of $N$ elastic binders and show how the presence of long-range interactions allows the system to recover the quasi-brittle behavior in the thermodynamic limit. In Sec. 4 we study the mean-field critical point in this system and link it a brittle-to-ductile transition. We then demonstrate that in this system, not only temperature but also the overall rigidity may affect the mode of failure. Our conclusions are summarized in Sec. 5 .

\section{MODEL OF A SINGLE BINDER}

We represent an elementary binding unit by a linear spring in series with a breakable element, see Fig. 1. For analytical simplicity, we model such an element as a 'fuse' with a piecewise quadratic 
potential

$$
U(X)= \begin{cases}\frac{\kappa_{p} X^{2}}{2}, & \text { if } X \leq L \\ \frac{\kappa_{p} L^{2}}{2}, & \text { if } X>L,\end{cases}
$$

where $\kappa_{p}$ is the linear elastic modulus before breaking. When the coordinate $X$ reaches the threshold $L$, the bond breaks and under further stretching its energy remains constant and equal to $\kappa_{p} L^{2} / 2$. The total energy of the binder loaded in a hard device takes the form

$$
E(X, Y)=U(X)+\frac{\kappa}{2}(Y-X)^{2},
$$

where $\kappa$ is the elasticity of the parallel spring and $Y$ is the controlling parameter representing total elongation, see Fig. 1.

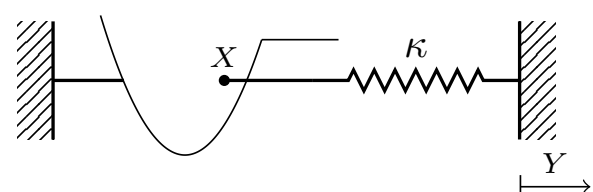

Figure 1. Mechanical representation of an individual binding element

It will be convenient to work with dimensionless variables. We set the breaking threshold $L$ as the reference length and introduce the dimensionless lengths $x=X / L, y=Y / L$ and energies $e=E / \kappa_{p} L^{2}, u=U / \kappa_{p} L^{2}$ and define the dimensionless parameter $\lambda=\kappa / \kappa_{p}$ controlling the effective toughness of the binder. Then the non-dimensional energy reads

$$
e(x, y)=u(x)+\frac{\lambda}{2}(y-x)^{2}
$$

where now $y$ is the controlling parameter characterizing the hard loading device (Fig. 2). In equilibrium $\partial e(x, y) / \partial x=0$ and the internal variable $x$ can be eliminated giving two locally stable branches

$$
e(y)= \begin{cases}\frac{\lambda}{\lambda+1} \frac{y^{2}}{2}, & \text { for } y \leq \frac{\lambda+1}{\lambda}, \\ \frac{1}{2}, & \text { for } y>1\end{cases}
$$

The ensuing double-valued force-elongation relation $t(y)=\partial e(y) / \partial y$ takes the form

$$
t= \begin{cases}\frac{\lambda}{\lambda+1} y, & \text { for } y \leq \frac{\lambda+1}{\lambda} \\ 0, & \text { for } y>1\end{cases}
$$

Observe that at $y_{*}=\sqrt{\frac{\lambda+1}{\lambda}}$, the ground state switches from unbroken to broken; however, both states can coexist as metastable in the interval $y \in[1,1+1 / \lambda]$. Therefore, the role of the series spring characterized by the parameter $\lambda$ is to ensure the mechanical bi-stability of the individual binders. In Fig. 3, we show how the size of the bi-stability domain depends on the parameters $\lambda$ and $y$. Note that in the limit $\lambda \rightarrow \infty$ the bonds behave as simple 'fuses', while at $\lambda \rightarrow 0$ the range of bi-stability where the bonds behave as mechanical snap-springs diverges. 


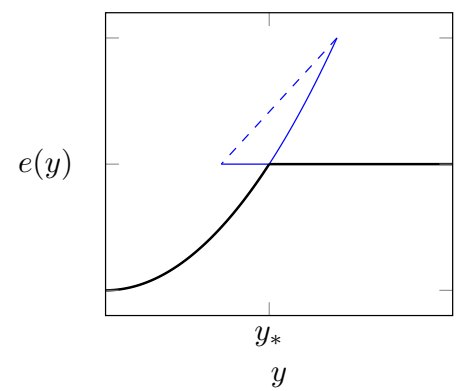

(a)

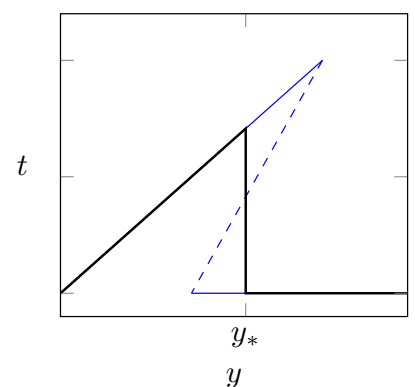

(b)

Figure 2. Mechanical response of a single binder in a hard device. Solid black lines, global minimum; colored lines, metastable states. Parameters: $\lambda=1$.
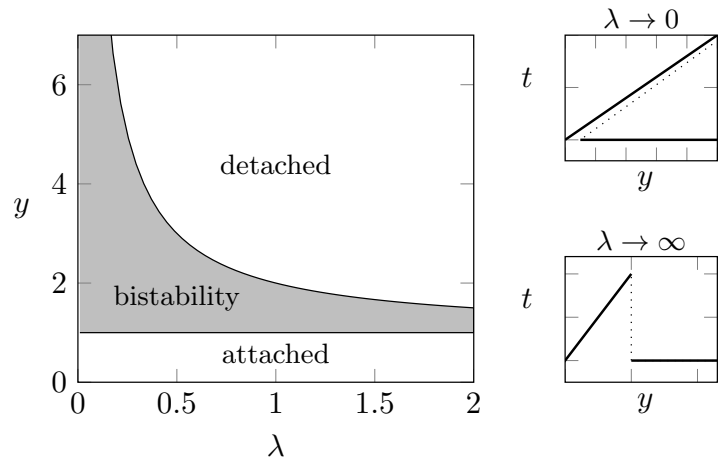

Figure 3. The dependence of the boundaries of the domain of metastability for a single binder on the loading $y$ and the stiffness $\lambda$

Consider now the same system at nonzero temperature. To compute the averaged mechanical response, we need to know the free energy as a function of the loading parameter

$$
\mathcal{F}(\beta, y)=-\frac{1}{\beta} \log \mathcal{Z}(y, \beta)
$$

Here $\mathcal{Z}(\beta, y)=\int_{-\infty}^{\infty} \exp [-\beta e(x ; y)] d x$ is the partition function, $\beta=\left(\kappa_{p} L^{2}\right) /\left(k_{b} T\right)$ is the nondimensional inverse temperature and $k_{b}$ is the Boltzmann constant. After a straightforward computation we obtain

$$
\begin{aligned}
\mathcal{Z}(\beta, y)=\sqrt{\frac{\pi}{2 \beta(1+\lambda)}} e^{-\frac{\lambda \beta}{2(1+\lambda)} y^{2}}\left\{\operatorname { e r f } \left[\sqrt{\left.\left.\frac{\beta}{2(1+\lambda)}(1+\lambda-\lambda y)\right]+1\right\}+}\right.\right. & \sqrt{\frac{\pi}{2 \beta \lambda}} e^{-\frac{\beta}{2}}\left\{\operatorname{erf}\left[\sqrt{\frac{\beta \lambda}{2}}(y-1)\right]+1\right\},
\end{aligned}
$$

where $\operatorname{erf}(x)=\frac{2}{\sqrt{\pi}} \int_{0}^{x} e^{-t^{2}} d t$. The equilibrium tension is then obtained by differentiation $t(\beta, y)=$ $\partial \mathcal{F}(\beta, y) / \partial y$.

The typical force-elongation relations are illustrated in Fig. 4. Note that the metastability has disappeared and that the reversible transition from attached to detached state is now gradual at any 
nonzero temperature. This is not surprising because in equilibrium the metastability disappears, for instance, at zero temperature our approach becomes equivalent to finding the global minimum of the energy. One can say that a single binder exhibits 'ductile' behavior while becoming 'brittle' only at zero temperature. This will also be the case in the standard FBM model with $N$ elements and controlled elongation because in such model individual binders are effectively independent. In the next Section, we show that 'brittleness' at finite temperature can be recovered in a hard device if individual binding elements interact sufficiently strong and can, therefore, bind (zip) and unbind (unzip) cooperatively.

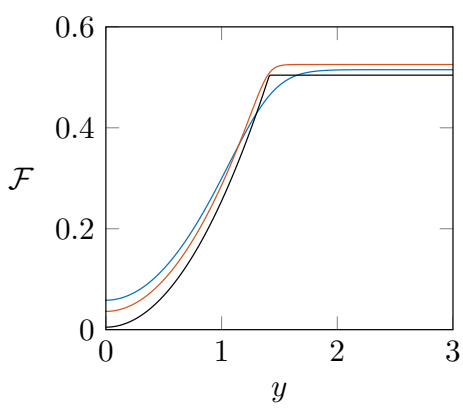

(a)

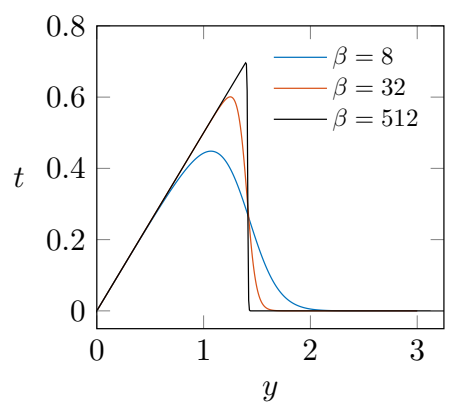

(b)

Figure 4. Mechanical response of a single bond in a hard device (imposed $y$ ) at several values of temperature for $\lambda=1$ : (a) Free energy; (b) Averaged tension.

\section{PARALLEL BUNDLE OF $N$ INTERACTING BINDERS}

Consider now $N$ parallel binders attached to a pair of rigid backbones. Assume further that the system is loaded through an external spring with stiffness $\kappa_{f}$ that characterizes the elasticity of the environment, see also $[36,51,52]$. The total energy of the system is

$$
E=\sum_{i=1}^{N}\left[U_{i}\left(X_{i}\right)+\frac{\kappa}{2}\left(Y-X_{i}\right)^{2}\right]+\frac{\kappa_{f}}{2}(Z-Y)^{2},
$$

where $Z$ is the total elongation which is the new controlling parameter, see Fig. 5 . In dimensionless variables the energy (8) reads

$$
v(\boldsymbol{x}, y, z)=\sum_{i=1}^{N}\left[u_{i}\left(x_{i}\right)+\frac{\lambda}{2}\left(y-x_{i}\right)^{2}+\frac{\Lambda}{2}(z-y)^{2}\right],
$$

where $\Lambda=\kappa_{f} /\left(N \kappa_{p}\right)$ is a new dimensionless parameter of the problem characterizing the degree of the cooperation between individual bonds: At $\Lambda=\infty$, the binders do not interact and at $\Lambda=0$ the system is effectively loaded in a soft device and the detached state disappears. The interesting cases are then at $\Lambda$ large but not too large.

Since we are interested in reversible equilibrium behavior, the task is again to compute the partition function

$$
\mathcal{Z}(\beta, z)=\int_{\Omega} \exp [-\beta v(\boldsymbol{x} ; y ; z)] d \boldsymbol{x} d y
$$




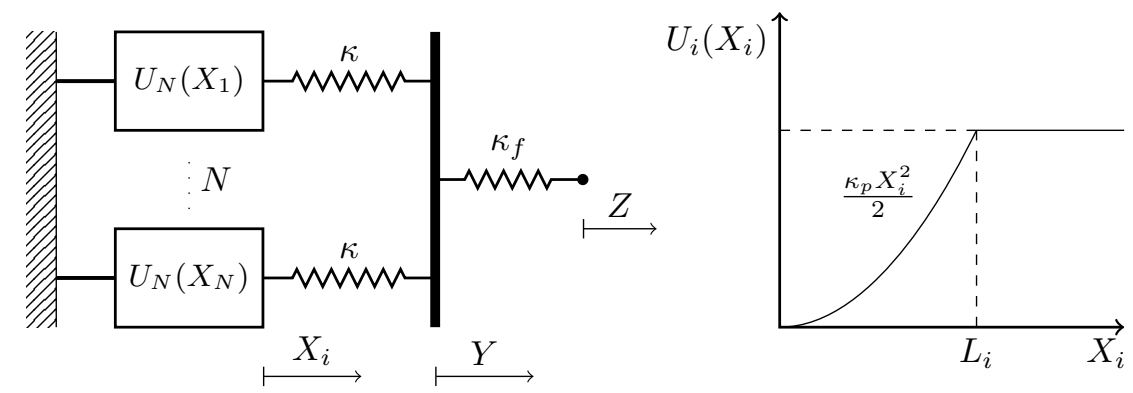

Figure 5. Mechanical model of the bundle and the potential energy of a single bond.

where now $\Omega=\mathbb{R}^{N} \times \mathbb{R}$. Given that the problem is permutationally invariant, we may say that in a given configuration the first $N p$ bonds, $x_{1}, \ldots, x_{N p}$, are attached, while the remaining bonds $x_{N p+1}, \ldots, x_{N}$ are detached. We can then write

$$
v\left(x_{1}, \ldots, x_{N}, y, z, p\right)=N \frac{\Lambda}{2}(z-y)^{2}+\sum_{i=1}^{N p} V_{1}\left(x_{i}, y\right)+\sum_{i=N p+1}^{N} V_{0}\left(x_{i}, y\right)
$$

Here $V_{1}\left(x_{i}, y\right)=\frac{x_{i}^{2}}{2}+\frac{\lambda}{2}\left(y-x_{i}\right)^{2}$ with $x_{i} \leq 1$, and $V_{0}\left(x_{i}, y\right)=\frac{1}{2}+\frac{\lambda}{2}\left(y-x_{i}\right)^{2}$ with $x_{i}>1$. Since for each $p$ there are $\left(\begin{array}{c}N \\ N p\end{array}\right)=\frac{N !}{(N p) !(N-N p) !}$ ways of choosing $N p$ bonds, we can thus write the expression for the marginal partition function at fixed $p$ and $y$

$$
\begin{aligned}
\mathcal{Z}(\beta, z, y, p)=\left(\begin{array}{c}
N \\
N p
\end{array}\right) e^{-\beta N \frac{\Lambda}{2}(z-y)^{2}} & \int_{-\infty}^{1} \ldots \int_{-\infty}^{1} \exp \left[-\beta \sum_{i=1}^{N p} V_{1}\left(x_{i}, y\right)\right] d x_{1} \ldots d x_{N p} \\
& \int_{1}^{\infty} \cdots \int_{1}^{\infty} \exp \left[-\beta \sum_{i=N p+1}^{N} V_{0}\left(x_{i}, y\right)\right] d x_{N p+1} \ldots d x_{N} .
\end{aligned}
$$

After some straightforward manipulations we obtain

$$
\mathcal{Z}(\beta, z, y, p)=\exp \left[-\beta N\left(\bar{v}(y, p, z)-\frac{1}{\beta} \bar{S}(\beta, y, p)-\frac{1}{\beta N} \ln \left(\begin{array}{c}
N \\
N p
\end{array}\right)\right)\right]
$$

where

$$
\bar{v}(z, y, p)=\frac{\Lambda}{2}(z-y)^{2}+p \frac{\lambda}{2(1+\lambda)} y^{2}+(1-p) \frac{1}{2},
$$

and

$$
\begin{array}{r}
\bar{S}(\beta, y, p)=p \ln \left[\sqrt{\frac{\pi}{2 \beta(1+\lambda)}}\left\{\operatorname{erf}\left[\sqrt{\frac{\beta}{2(1+\lambda)}}(1+\lambda-\lambda y)\right]+1\right\}\right] \\
+(1-p) \ln \left[\sqrt{\frac{\pi}{2 \beta \lambda}}\left\{\operatorname{erf}\left[\sqrt{\frac{\beta \lambda}{2}}(y-1)\right]+1\right\}\right]
\end{array}
$$




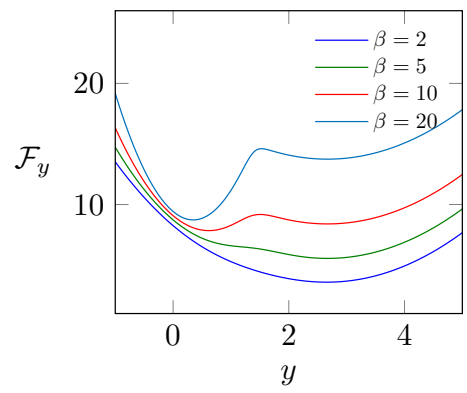

(a)

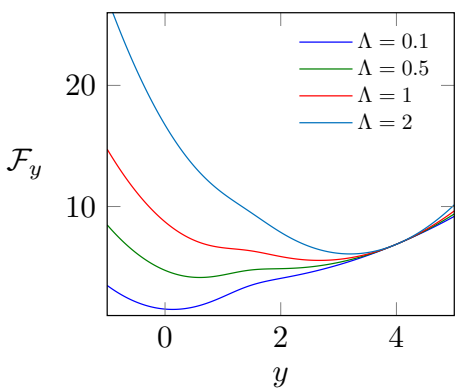

(b)

Figure 6. Free energy (16) for $\lambda=1$ and $z=4$. In (a) we fix $\Lambda=1$ and vary $\beta$, and in (b) we fix $\beta=5$ and vary $\Lambda$.

To eliminate the variable $p$, we need to sum over all its possible values.

$$
\mathcal{Z}(\beta, z, y)=\sum_{N p=0}^{N} \mathcal{Z}(\beta, z, y, p)
$$

Note that in the thermodynamic limit $N \rightarrow \infty$ we can replace the sum over $p$ by an integral over the interval $[0,1]$. We then obtain the marginal (fixed $y$ ) partition function

$$
\mathcal{Z}(\beta, z, y)=\exp \left[-\beta N\left(\hat{v}(y, z)-\frac{1}{\beta} g(\beta, y)\right)\right]
$$

where

$$
\hat{v}(y, z)=\frac{\Lambda}{2}(z-y)^{2}
$$

and

$$
\begin{array}{r}
g(\beta, y)=\ln \left[\sqrt{\frac{\pi}{2 \beta(1+\lambda)}} e^{\frac{-\lambda \beta}{2(1+\lambda)} y^{2}}\left\{\operatorname{erf}\left[\sqrt{\frac{\beta}{2(1+\lambda)}}(1+\lambda-\lambda y)\right]+1\right\}\right. \\
\left.+\sqrt{\frac{\pi}{2 \beta \lambda}} e^{-\frac{\beta}{2}}\left\{\operatorname{erf}\left[\sqrt{\frac{\beta \lambda}{2}}(y-1)\right]+1\right\}\right]
\end{array}
$$

The corresponding marginal free energy

$$
\mathcal{F}_{y}(\beta, z, y)=\hat{v}(y, z)-\frac{1}{\beta} g(\beta, y)
$$

is illustrated in Fig. 6.

The most striking feature of this free energy is its non-convexity at sufficiently small temperatures (large $\beta$ ) and sufficiently strong coupling between individual binders (small $\Lambda$ ). This is a result of the presence of long-range interactions in the system [53]. The two macroscopic energy wells in the parameter range of non-convexity suggest a synchronization of individual binders and indicate the possibility of highly coherent, collective transitions between attached (zipped) and detached (unzipped) states. In Fig. 7 we show the location of the domain of non-convexity of 


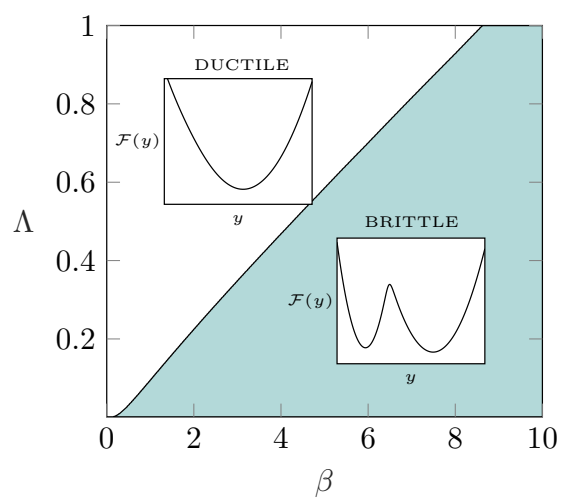

Figure 7. Phase diagram showing different convexity domains of the marginal free energy (16).

$\mathcal{F}_{y}(y)$ in the plane $(\beta, \Lambda)$. In the next section, it will be associated with 'brittle' behavior, while the behavior of the system in the domain of convexity of $\mathcal{F}_{y}(y)$ will be interpreted as ductile.

To argue about the macroscopic behavior, we still need to integrate over the order parameter $y$ and compute the equilibrium partition function $\mathcal{Z}(\beta, z)=\int_{-\infty}^{\infty} \mathcal{Z}(\beta, z, y) d y$. Using the Laplace method, we obtain $\mathcal{Z}(\beta, z) \sim \exp \left[-N \beta \mathcal{F}_{y}\left(y_{0}(\beta, z), \beta, z\right)\right]$ where $y_{0}(\beta, z)$ is the global minimum of the function $\mathcal{F}_{y}(y, \beta, z)$. We can then compute the free energy density $\mathcal{F}(\beta, z)=\mathcal{F}_{y}\left(y_{0}(\beta, z), \beta, z\right)$ and study the equilibrium tension-elongation $t(\beta, z)=\partial \mathcal{F}(\beta, z) / \partial z$. The nontrivial behavior of the ensuing mechanical response is due to the double well nature of the marginal free energy density $\mathcal{F}_{y}(y)$ and the resulting discontinuous dependence of the function $y_{0}(\beta, z)$ on the loading parameter $z$.

\section{BRITTLE-TO-DUCTILE TRANSITION}

Our main results are summarized in Fig. 8 showing the mechanical response of the system in the thermodynamic limit $N \rightarrow \infty$. Depending on the point in the parameter space (Fig. 7 ) the behavior of the system can be qualified as brittle or ductile. In the ductile regime, the response is continuous in the whole range of loading, in the sense that the zipped state becomes unzipped gradually as the loading parameter $z$ increases from zero to infinity. In such systems, the average fraction of attached binders $p$ changes continuously under loading and the tension $t$ decreases progressively. Instead, in the brittle regime, the system undergoes at some $z=z_{*}(\beta, \Lambda)$ a singular macroscopic transition from zipped to unzipped state with an abrupt drop of tension. The transition is associated with a jump in the number of attached binders which means that it is a collective phenomenon and that behind it is a synchronized switch of the significant fraction of binding elements from one macro energy well to another. Brittle and ductile regimes are separated in Fig. 7 by a line of critical points which can also be interpreted as the boundary of a second-order phase transition. The situation is formally similar to what is observed in mean-field spin systems describing the transition from para to ferromagnetism $[54,55]$. In the brittle (ferromagnetic) phase, the marginal free energy $\mathcal{F}(y)$ is non-convex for some $z$ and the equilibrium free energy $\mathcal{F}(z)$ has a singularity at $z=z_{*}$, while in the ductile (paramagnetic) phase the marginal free energy is always convex and the equilibrium free energy is analytic. We emphasize, however, that the discontinuous (brittle) tension-elongation response associated with the singularity of the equilibrium free energy is possible only in the thermodynamic limit. In other words, at finite $N$ the free energy is always smooth, and the mechanical response is always ductile even though it may be arbitrarily sharp. 
(a)

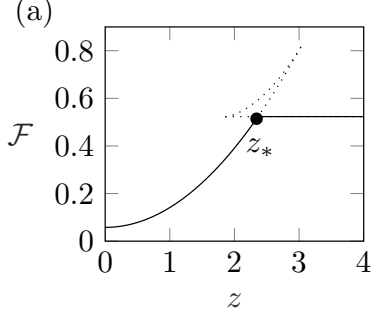

(d)

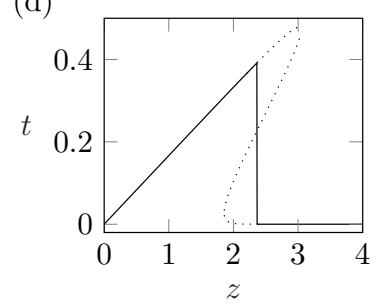

(g)

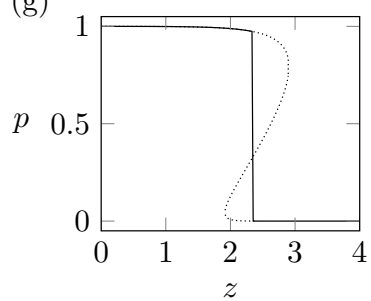

BRITTLE (b)

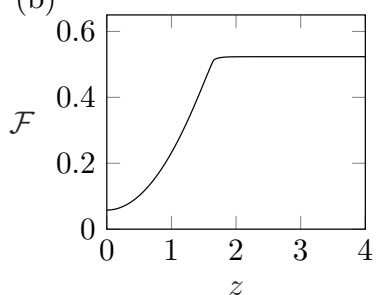

(e)

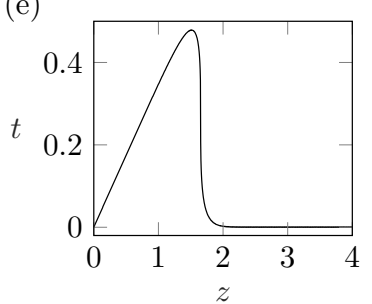

(h)

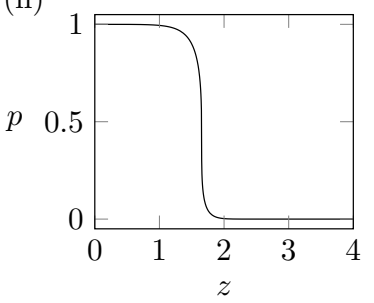

CRITICAL

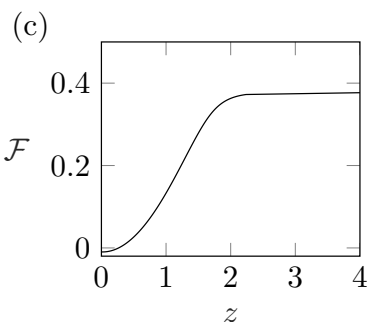

(f)

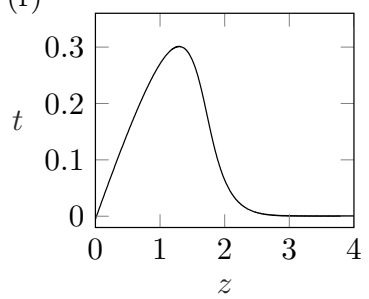

(i)

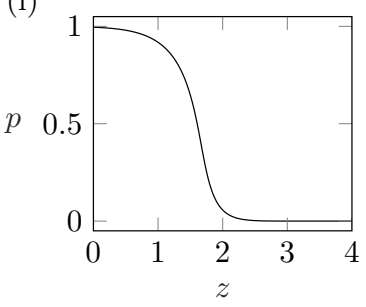

DUCTILE

Figure 8. Mechanical response of system in ductile, critical and brittle regimes. Brittle: $\beta=8, \lambda=1$, $\Lambda=0.25$. Critical: $\beta=8.62, \lambda=1, \Lambda=1$. Ductile: $\beta=4, \lambda=1, \Lambda=1$.

In Fig. 9, we illustrate a peculiar effect of temperature on the behavior of the system in the brittle regime. Thus, with temperature increasing at the fixed elongation $z$ one would expect to see an abrupt thermal unzipping (denaturation) when the temperature reaches a particular threshold and the bonds collectively disassociate. This is indeed how the system behaves in general. However, we also see in Fig. 9 that some partial bonding serves as a precursor of the eventual macroscopic unzipping. The detailed study of this phenomenon, which can be associated with negative thermal expansion, will be presented elsewhere.
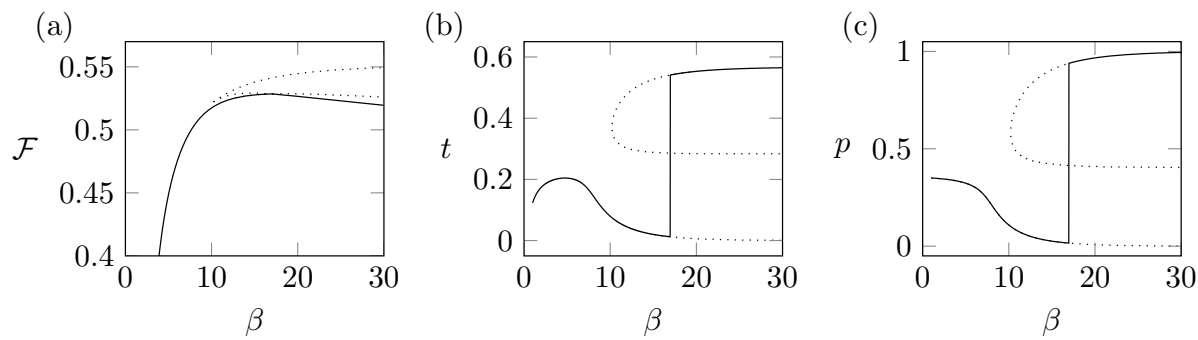

Figure 9. Mechanical response of the system at a fixed elongation $z=1.7$ and varying inverse temperature $\beta$. parameters: $\lambda=1, \Lambda=1$. Solid lines represent equilibrium behavior, dotted lines - metastable branches.

Suppose now that both the elongation $z$ and the temperature $\beta$, are fixed. In this case, the discontinuous transition between the zipped configuration with $p \sim 1$ and the unzipped configura- 
tion with $p \sim 0$ can be induced by changing the 'internal' stiffness $\lambda$, see Fig. 10c, or the 'external' stiffness $\Lambda$, see Fig. 11c. For this behavior, which may be of a particular interest in biological applications, e.g., $[56,57]$, to be relevant the temperature of the system must be, of course, sufficiently low.
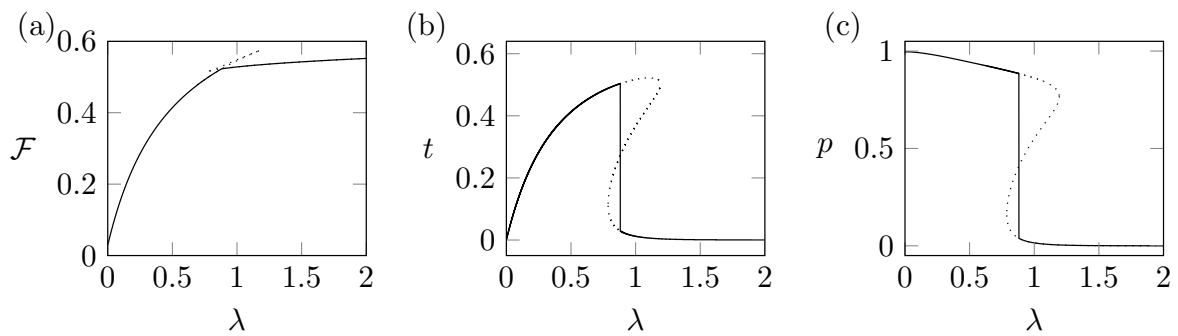

Figure 10. Mechanical response of the system at a fixed elongation $z=\sqrt{3}$, temperature $\beta=15$ and varying internal stiffness $\lambda$. Parameters: $\Lambda=1$. Solid lines represent the equilibrium response, and dotted lines represent metastable branches.
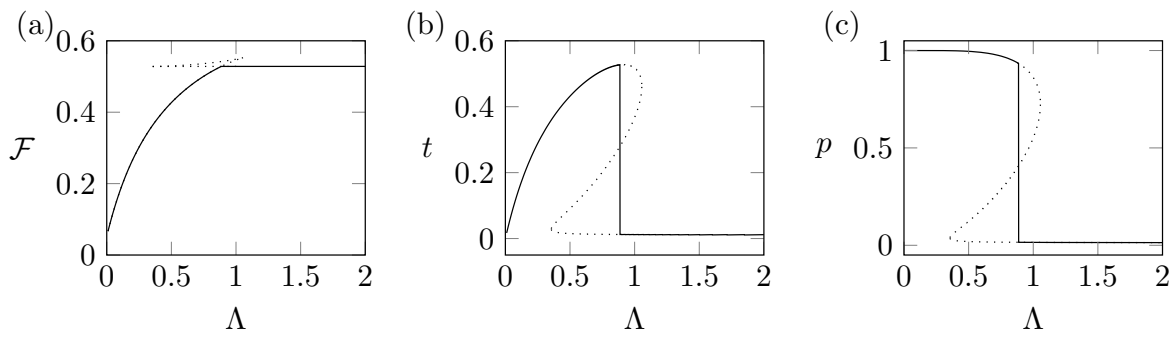

Figure 11. Mechanical response of the system at a fixed elongation $z=\sqrt{3}$, temperature $\beta=15$, and varying external stiffness $\Lambda$. Parameters: $\lambda=1$. Solid lines represent the equilibrium response, and dotted lines represent metastable branches.

\section{CONCLUSIONS}

We presented a mean-field model of displacement controlled unzipping at finite temperature showing that the interplay between the rigidity and temperature may drastically change the overall response of the system from ductile, where the debonding is gradual, to brittle, where the debonding is discontinuous.

The study of a single binder elucidated the role played by the internal stiffness introducing bi-stability and allowing the attached and the detached states to coexist and intermingle in time. The continuous 'phase switching', however, destroys the snap-through characteristics of a binder at any nonzero temperature.

We then showed that one recovers the snap-through behavior in an infinite system of such binders interacting through a mean field. In such a system, the interaction effectively 'freezes' the binders in one of the macroscopic free energy wells allowing only a cooperative switch between the attached and the detached states. We constructed a phase diagram in the temperature-rigidity space showing that a line of critical points separates brittle (cooperative) and ductile (noncooperative) behaviors. We, therefore, interpreted the brittle-to-ductile transition in this system as a second-order phase transition. 
One of our important observations is that the discontinuous unzipping in such systems can be induced by tuning the overall rigidity. As it has been recently shown, in biological systems this kind of tuning can be achieved actively [57]. Our study then suggests that active rigidity manipulation may be an important factor controlling cell adhesion and may even facilitate filamental cross-linking during the reorganization of the cytoskeleton.

\section{ACKNOWLEDGMENTS}

The authors thank R. Garcia-Garcia for helpful discussions. H.B.R. was supported by a Ph.D. fellowship from Ecole Polytechnique; L. T. was supported by the French Government under the Grants ANR-10-IDEX-0 0 01-02 PSL and ANR-17-CE08-0 047-02.

[1] M. Kanninen and C. Popelar, Advanced Fracture Mechanics, Oxford engineering science series (Oxford University Press, 1985).

[2] S. S. Brenner, Journal of Applied Physics 33, 33 (1962).

[3] R. L. B. Selinger, Z.-G. Wang, and W. M. Gelbart, The Journal of Chemical Physics 95, 9128 (1991).

[4] R. F. Cook and E. G. Liniger, Journal of the American Ceramic Society 76, 1096 (1993).

[5] V. A. Petrov and A. N. Orlov, International Journal of Fracture 12, 231 (1976).

[6] X.-S. Xing, Engineering Fracture Mechanics 38, 1 (1991).

[7] V. Berdichevsky and K. C. Le, International Journal of Fracture 133, L47 (2005).

[8] K. K. a. A. S. Krausz, Fracture Kinetics of Crack Growth, 1st ed., Mechanical Behavior of Materials 1 (Springer Netherlands, 1988).

[9] Y. Pomeau, C. R. Acac. Sci. Paris Serie II, 553 (1992).

[10] S. Ciliberto, A. Guarino, and R. Scorretti, Physica D: Nonlinear Phenomena 158, 83 (2001).

[11] A. Politi, S. Ciliberto, and R. Scorretti, Phys. Rev. E 66, 026107 (2002).

[12] G. Bell, Science 200, 618 (1978).

[13] U. S. Schwarz and S. A. Safran, Rev. Mod. Phys. 85, 1327 (2013).

[14] E. Evans and K. Ritchie, Biophysical Journal 72, 1541 (1997).

[15] B. Chakrabarti and D. R. Nelson, The Journal of Physical Chemistry B 113, 3831 (2009).

[16] C. Hyeon and D. D. Thirumalai, Molecular Modeling at the Atomic Scale: Methods and Applications in Quantitative Biology , 141 (2014).

[17] R. K. Mishra, T. Modi, D. Giri, and S. Kumar, The Journal of Chemical Physics 142, 174910 (2015).

[18] A. E. Bergues-Pupo, J. M. Bergues, F. Falo, and A. Fiasconaro, The European Physical Journal E 38, 41 (2015).

[19] T. Strunz, K. Oroszlan, R. Schäfer, and H.-J. Güntherodt, Proceedings of the National Academy of Sciences 96, 11277 (1999).

[20] J. Helenius, C.-P. Heisenberg, H. E. Gaub, and D. J. Muller, Journal of Cell Science 121, 1785 (2008).

[21] J. Friedrichs, J. Helenius, and D. J. Muller, Nature Protocols 5, 1353 (2010).

[22] D. Gonzalez-Rodriguez, L. Bonnemay, J. Elgeti, S. Dufour, D. Cuvelier, and F. Brochard-Wyart, Soft Matter 9, 2282 (2013).

[23] B. Hogan, A. Babataheri, Y. Hwang, A. I. Barakat, and J. Husson, Biophysical Journal 109, 209 (2015).

[24] R. L. B. Selinger, Z.-G. Wang, W. M. Gelbart, and A. Ben-Shaul, Phys. Rev. A 43, 4396 (1991).

[25] S. Roux, Phys. Rev. E 62, 6164 (2000).

[26] R. Scorretti, S. Ciliberto, and A. Guarino, EPL (Europhysics Letters) 55, 626 (2001).

[27] M. J. Alava, P. K. V. V. Nukala, and S. Zapperi, Advances in Physics 55, 349 (2006).

[28] A. Virgilii, A. Petri, and S. R. Salinas, Journal of Statistical Mechanics: Theory and Experiment 2007, P04009 (2007).

[29] N. Yoshioka, F. Kun, and N. Ito, Phys. Rev. E 91, 033305 (2015).

[30] T. Erdmann and U. S. Schwarz, Phys. Rev. Lett. 92, 108102 (2004). 
[31] M. Peyrard and A. R. Bishop, Phys. Rev. Lett. 62, 2755 (1989).

[32] M. Manghi and N. Destainville, Physics Reports 631, 1 (2016), physics of base-pairing dynamics in DNA.

[33] A. Vologodskii and M. D. Frank-Kamenetskii, Physics of Life Reviews (2017).

[34] T. Erdmann, P. J. Albert, and U. S. Schwarz, The Journal of Chemical Physics 139, 175104 (2013).

[35] M. Caruel and L. Truskinovsky, Reports on Progress in Physics 81, 036602 (2018).

[36] U. Seifert, Phys. Rev. Lett. 84, 2750 (2000).

[37] T. Erdmann and U. Schwarz, Eur. Phys. J. E 22, 123 (2007).

[38] T. Erdmann and U. S. Schwarz, Biophysical Journal 91, L60 (2006).

[39] A. F. Huxley and R. M. Simmons, Nature 233, 533 (1971).

[40] U. Seifert, EPL (Europhysics Letters) 58, 792 (2002).

[41] H. Kramers, Physica 7, 284 (1940).

[42] P. Hänggi, P. Talkner, and M. Borkovec, Rev. Mod. Phys. 62, 251 (1990).

[43] S. Pradhan, A. Hansen, and B. K. Chakrabarti, Reviews of Modern Physics 82, 499 (2010).

[44] A. Hansen, P. Hemmer, and S. Pradhan, The Fiber Bundle Model: Modeling Failure in Materials, Statistical Physics of Fracture and Breakdown (Wiley, 2015).

[45] R. Batra, Elements of Continuum Mechanics, AIAA education series (American Institute of Aeronautics \& Astronautics, 2006).

[46] H. J. Kreuzer and S. H. Payne, Phys. Rev. E 63, 021906 (2001).

[47] M. Caruel and L. Truskinovsky, Journal of the Mechanics and Physics of Solids 109, 117 (2017).

[48] A. Fuhrmann and A. J. Engler, Biophysical Journal 109, 57 (2015).

[49] D. E. Discher, P. Janmey, and Y.-l. Wang, Science 310, 1139 (2005).

[50] T. Yeung, P. C. Georges, L. A. Flanagan, B. Marg, M. Ortiz, M. Funaki, N. Zahir, W. Ming, V. Weaver, and P. A. Janmey, Cell Motility 60, 24 (2004).

[51] F. Jülicher and J. Prost, Phys. Rev. Lett. 75, 2618 (1995).

[52] A. Delaplace, S. Roux, and G. P. jaudier Cabot, International Journal of Solids and Structures 36, 1403 (1999).

[53] A. Campa, T. Dauxois, and S. Ruffo, Physics Reports 480, 57 (2009).

[54] M. Caruel and L. Truskinovsky, Phys. Rev. E 93, 062407 (2016).

[55] R. Balian, From microphysics to macrophysics: methods and applications of statistical physics, Theoretical and mathematical physics (Springer Science \& Business Media, 2007).

[56] G. Puglisi and L. Truskinovsky, Phys. Rev. E 87, 032714 (2013).

[57] R. Sheshka, P. Recho, and L. Truskinovsky, Phys. Rev. E 93, 052604 (2016). 